\title{
Imperforate hymen - a rare cause of acute abdomen in adolescent girls: a case report and literature review
}

Mehmet Kulhan, Nur Gozde Kulhan, Umit Nayki, Cenk Nayki, Nahit Ata

Department of Obstetrics and Gynecology, School of Medicine, Erzincan University, Erzincan, Turkey

Submitted: 26 February 2017

Accepted: 14 March 2017

Arch Med Sci Civil Dis 2017; 2: e59-e61

DOI: https://doi.org/10.5114/amscd.2017.66839

Copyright $\odot 2017$ Termedia \& Banach

As is well known, Müllerian duct anomalies are rare (0.001-10\%) in the community. Among them, the most common type is imperforate hymen $(0.1-0.01 \%)[1,2]$. Hymenal anomalies are derived from incomplete degeneration of the central portion of the hymen. These anatomic variants include imperforate, microperforate, septate, and cribriform hymens. An imperforate hymen is one of the most common obstructive lesions of the female genital tract. At birth, infants may have a bulging introitus due to mucocolpos from vaginal secretions stimulated by maternal estradiol. Otherwise, there is an undiagnosed imperforate hymen in the newborn, the mucus will be reabsorbed and the child usually remains asymptomatic until menarche. After menarche, the adolescent girl may present with a history of cyclic abdominal or pelvic pain and hematocolpos that leads to a hymenal membrane with bluish discoloration. Marked distension of the vagina may also result in back pain, pain with defecation, or difficulties in urination. In this paper we present a case of a patient admitted to the emergency department with acute abdominal pain, diagnosed as imperforate hymen after examination.

A 13-year-old girl was admitted to the emergency department with severe lower abdominal colic pain and concomitant anorexia that had lasted for 3 months. There was no history of constipation, diarrhea, vomiting, fever or any complaints related to the urinary system. Vital findings were normal in her physical examination. However, suprapubic fullness and abdominal pain were observed as well as positive rebound and defense. Laboratory findings were as follows: $\beta$-human chorionic gonadotropin $(\beta-\mathrm{hCG})<0.100 \mathrm{MIU} / \mathrm{ml}$, leukocytes: $11000 / \mathrm{mm}^{3}$, hematocrit: $43.2 \%$, hemoglobin ( $\mathrm{Hb}$ ): $13.4 \mathrm{~g} / \mathrm{dl}$, thrombocytes: $265000 / \mathrm{mm}^{3}$ in blood, while C-reactive protein (CRP) and tumor marker concentrations were within normal ranges. Urinalysis was normal. In her physical examination, secondary sex characters were detected to be developed (Tanner stage 4). She was referred to the pediatric surgery clinic, and the rectal examination performed by the pediatric surgeon revealed a soft, $10 \mathrm{~cm}$ large mass exerting pressure on the rectum. After the pelvic mass was confirmed by abdominal ultrasonography, she was referred to our gynecology clinic. In the gynecological examination, absence of the hymenal opening, and a swollen hymen with purple highlights which closed the vagina by forming a curtain around it were observed (Figure 1). In the repeated ultrasonographic examination, a hypoechoic cystic formation with smooth contours, approximately $13 \times 7 \mathrm{~cm}$ in diameter, was viewed along the pelvic midline (Figure 2). The pelvic magnetic resonance im-

\author{
Corresponding author: \\ Mehmet Kulhan \\ Department of Obstetrics \\ and Gynecology \\ School of Medicine \\ Erzincan University \\ 24030 Erzincan, Turkey \\ Phone: +90 5054918090 \\ E-mail: Mehmet_kulhan@ \\ yahoo.com
}




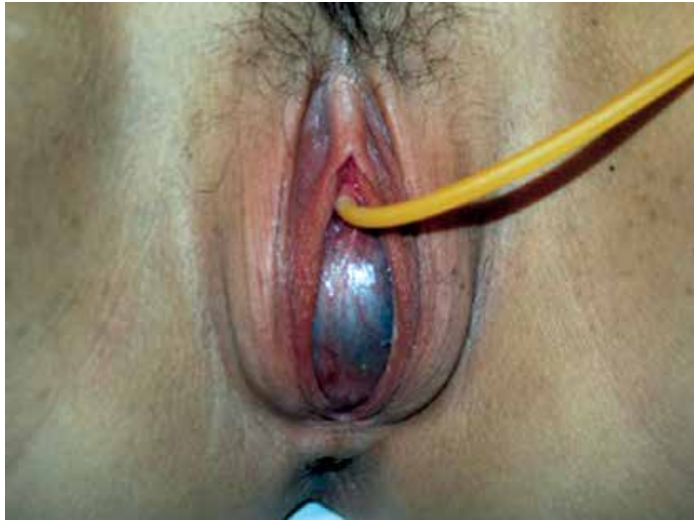

Figure 1. Photograph showing the bulging hymen with the collection of menstrual blood

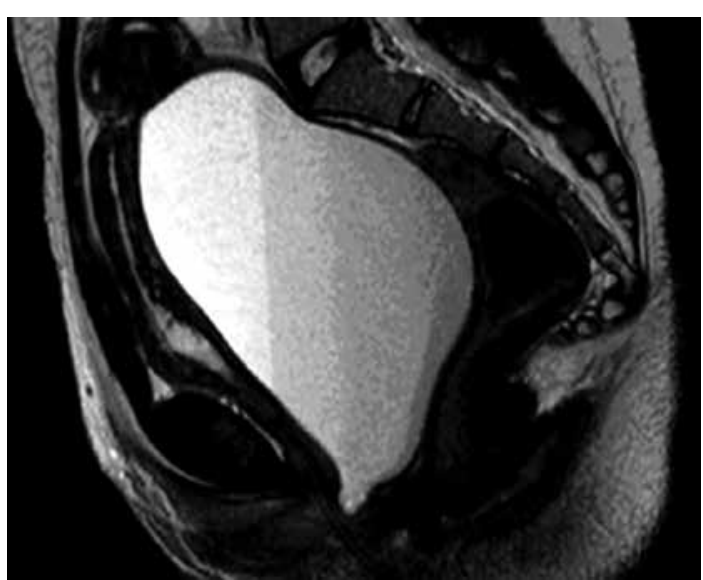

Figure 3. Magnetic resonance imaging scans showing the fluid indicative of blood products

aging (MRI) showed an appearance consistent with hematocolpos (Figure 3). Bilateral ovaries and uterus were normal. The urinary system was found to be normal after the intravenous pyelography (IVP). After all of these procedures, the patient was diagnosed with imperforate hymen, and hymenotomy was planned. In hymenotomy, an elliptical incision was made in the membrane close to the hymenal ring, followed by evacuation of the obstructed material under general anesthesia, and approximately 1000 cc of tarry menstrual blood was aspirated. Extra-hymenal tissue was excised using electrocautery to create a normal sized orifice, and the vaginal mucosa was sutured to the hymenal ring using 3-0 vicryl to prevent adhesion and recurrence of the obstruction. The patient was discharged on the next day without any problem. In her control examination after 1.5 months, no problem was noted and she was observed to have menstruated. The patient was followed regularly every month for 1 year.

Imperforate hymen is a rare but serious cause of abdominal pain in female adolescents. It is observed in approximately 1 in 2000 females [1-3]. Hymen is an embryological remnant of mesoder-

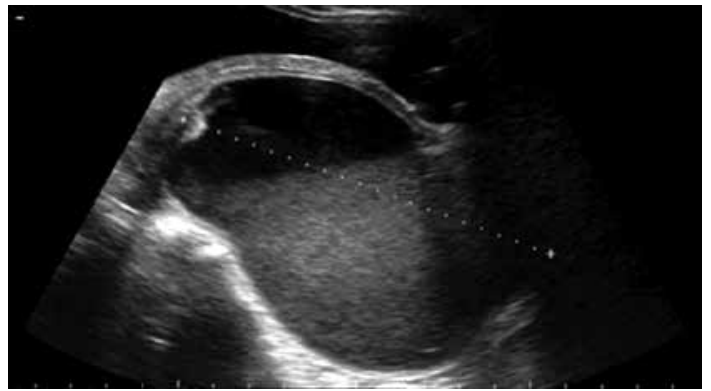

Figure 2. Ultrasound scan showing fluid-filled uterus

mal tissue that normally perforates during the later stages of embryonal development. The hymen is called imperforate if there is no perforation of this membrane. The reason for non-perforation of this membrane is unknown.

The most common symptoms of an imperforate hymen are cyclical abdominal pain and urinary retention, usually presenting between the ages of 13 and 15 years (when menarche occurs) $[4,5]$. There is primary amenorrhea but secondary sex characteristics are well developed. Because the vaginal outflow is obstructed by the non-perforated hymen, menstrual blood accumulates in the vagina (hematocolpos) and the uterus (hematometra). This may lead to mechanical effects on the urethra, bladder, intestines or pelvic blood vessels that can result in urinary retention, constipation or edema of the legs [4, 6]. Irritation of the sacral plexus or nerve roots can cause lower back pain [5] Problems with intercourse are rarely mentioned, probably because most of the patients are still sexually inactive [7].

Some symptoms of appendicitis are similar to those of an imperforate hymen, and there are cases where groundless appendectomies have been performed [8]. In addition, there is often an initial diagnosis of infection of the bladder, nephrolithiasis or abdominal tumor, which leads to unnecessary examinations and treatments. History and physical examination are frequently incomplete [2]. One should always consider an imperforate hymen if there is a discrepancy between the Tanner stage and menarcheal status [2].

Repair of the hymen can be performed at any age; however, the repair is facilitated if the tissues have undergone estrogen stimulation. Therefore, surgery is ideal in the newborn, postpubertal, or premenarchal age. Surgical repair under anesthesia consists of an elliptical incision in the membrane close to the hymenal ring followed by evacuation of the obstructed material. Extra-hymenal tissue is excised using electrocautery to create a normal sized orifice, and the vaginal mucosa is sutured to the hymenal ring using 3-0 or 4-0 vicryl or chromic sutures to prevent adhesion and recurrence of the obstruction. The complications of hymenectomy are bleeding, scarring and stenosis of 
the vaginal opening [9]. Incomplete fenestration of the hymenal opening (microperforate, septate, or cribriform) is often asymptomatic. Patients may require gynecologic evaluation because of inability to insert tampons, douches, or vaginal creams, or because of difficulty with coitus.

Treatment of microperforate, septate, and cribriform hymens involves resection of the excess hymenal tissue to create a functional hymenal ring, as described above. The excess hymenal tissue is excised by electrocautery, and interrupted sutures are placed to reapproximate the tissue.

\section{Conflict of interest}

The authors declare no conflict of interest.

\section{References}

1. Parazzini F, Cecchetti G. The frequency of imperforate hymen in Northern Italy. Int J Epidemiol 1990; 19: 763-4.

2. Posner JC, Spandorfer PR. Early detection of imperforate hymen prevents morbidity from delays in diagnosis. Pediatrics 2005; 115: 1008-12.

3. Dane C, Dane B, Erginbas M, et al. Imperforate hymen - a rare cause of abdominal pain: two cases and review of the literature. J Pediatr Adolesc Gynecol 2007; 20: 245-7.

4. Hall DJ. An unusual case of urinary retention due to imperforate hymen. J Accid Emerg Med 1999; 16: 232-3.

5. Letts $M$, Haasbeek J. Hematocolpos as a cause of back pain in premenarchal adolescents. J Pediatr Orthop 1990; 10: 731-2.

6. Dickson CA, Saad S, Tesar JD. Imperforate hymen with hematocolpos. Ann Emerg Med 1985; 14: 467-9.

7. Liang CC, Chang SD, Soong YK. Long-term follow-up of women who underwent surgical correction for imperforate hymen. Arch Gynecol Obstet 2003; 269: 5-8.

8. Nazir Z, Rizvi RM, Qureshi RN, et al. Congenital vaginal obstructions: varied presentation and outcome. Pediatr Surg Int 2006; 22: 749-53.

9. Chang JW, Yang LY, Wang HH, et al. Acute urinary retention as the presentation of imperforate hymen. J Chin Med Assoc 2007; 70: 559-61. 